\title{
Time Evolution of Hurst Exponent: Czech Intraday Electricity Market Study
}

\author{
Juraj Čurpek*
}

\begin{abstract}
:
In this paper we analyse a temporal evolution of the Hurst exponent estimated on hourly returns of intraday electricity prices in the Czech Republic in 2017 and 2018. Firstly we used the log-returns with adjustments due to negative values, and secondly we employed the returns based on the area hyperbolic sine transformation. We implemented a sliding window technique in order to estimate the Hurst exponent using the Detrended Fluctuation Analysis method on subsamples with four distinct window sizes. According to the stylised facts of electricity, the spot prices and their corresponding logarithmic returns should be mean-reverting. Since the Czech intraday electricity market remains mostly unexplored, we examined this phenomenon on the intraday rather than on the spot market. Consequently, our analysis showed that the estimated values of Hurst exponent indicate a meanreverting process for time scales greater than 24 hours and a weakly mean-reverting process for the shorter time scales. There were a few exceptions, though, since our calculations have revealed the presence of a nearly random or even weakly persistent behaviour on the shorter time scales.
\end{abstract}

Key words: Hurst exponent; Detrended Fluctuation Analysis; electricity markets; intraday market.

JEL classification: C13, G10.

\section{Introduction}

In developed countries, electricity trading currently takes place on liberal and deregulated markets. Unlike other commodities, electricity is economically still not viable to store since there is no efficient and financially sensible way of storing it, even though electricity can actually be stored in batteries or through the use of pumped hydro storage dams. Moreover, at every moment the aggregate supply must meet the aggregate demand. Otherwise, shortages of electricity would occur at some places or the electricity grid would collapse. Therefore, the price of electricity should respect the transmission grid constraints and the market coupling in EU, which means the integration of two or more electricity markets from

Juraj Čurpek; University of Economics, Prague, Faculty of Finance and Accounting, Department of Banking and Insurance, W. Churchill Sq. 4, 13067 Prague 3, Czech Republic, <xcurj07@vse.cz>.

The article is processed as an output of a research project Vývojové trendy na finančnich trzích registered by the Grant Agency of University of Economics, Prague under the registration number F1/46/2019. 
different EU areas through the implicit cross-border allocation mechanism (Bergh et al., 2016). Moreover, the so-called intermittent renewable energy sources, mostly solar and wind generation units, have been recently widely used in many countries, and these strongly depend on weather conditions. Together with an increase in the capacity of the power grid, they have caused a higher volatility in the electricity load and price (Hong et al, 2016).

The presented paper focuses on the Czech wholesale intraday electricity market where electricity is traded between the producers and retailers or large consumers. Although there is a plethora of studies that deal with the spot (day-ahead) prices, amount of research covering the topic of intraday electricity prices is rather scarce and to this date none of it has dealt with the Hurst analysis of the intraday electricity prices or their returns, concentrating rather on forecasting. For instance, the intraday prices prediction was analysed by Monteiro et al. (2016) using artificial intelligence on the Iberian electricity market. They deduced that variables with the largest impact were the previous intraday prices and seasonal dummies. In the paper by Uniejewski et al. (2019), which focused on the forecasting and variable selection using the intraday prices transformed using the area (inverse) hyperbolic sine, it was concluded that the most important regressors were the day-ahead price for the same or nearby hour and the most recent intraday price. Regarding the Czech electricity market, so far there is only a single relevant research by Béreš (2016), who analysed the intraday market and its impact on the day-ahead counterpart with the conclusion that the forecast errors of the renewable (mainly solar) generation and load affect the intraday prices. Furthermore, the negative intraday prices are related to the unexpected surplus of the renewable generation or the negative demand shock and there is no connection with the negative day-ahead prices.

According to the stylised facts of electricity prices, the spot prices and also their returns are mean-reverting. However, this feature has not been explored for intraday prices or their respective returns, which is the goal of this paper. Application of the Hurst exponent analysis, which quantifies the long-term memory in time series, states that the mean-reverting process should have a certain value of the Hurst exponent, specifically between zero and 0.5 . Thus, we can estimate the timeevolution of the Hurst exponent for intraday electricity returns, which could indicate the potential presence of mean-reversion.

Relevant empirical studies that concentrated solely on the mean-reversion of the spot electricity prices found a weak-mean reversion (Simonsen, 2003) on the Nord Pool market. Meyer-Brandis et al. (2008) concluded that a daily spot electricity price on several European electricity markets is a sum of several mean-reverting processes with different speeds of mean reversion.

Regarding the empirical studies concerning the Hurst exponent of electricity prices, it was shown by Kristoufek et al. (2013) that hourly prices of electricity on the 
Czech market over the period 2009-2012 were mean-reverting with the scaling exponent $h \approx 1.1$, which corresponds to the Hurst exponent of $H \approx 0.1$. In another study (Weron, 2000), analysis of the logarithmic returns of average daily electricity prices on the California Power Exchange (CalPX) and Swiss Electricity Price (SWEP) from 1998 to 2000 has concluded with the similar results, i.e. that logarithmic returns are mean-reverting. In an extensive study of electricity prices predictability in the Canadian provinces of Alberta and Ontario and the US Mid-C market (Uritskaya et al., 2015) it was concluded that the original prices exhibited strong mean-reverting behaviour as well, which is in accordance with the stylised facts.

Since the number of studies on the Czech intraday market is limited and there are no actual studies concerning the Hurst exponent analysis on the Czech intraday electricity market, the aim of this paper is to examine this matter and fill the gap.

\section{Electricity Prices}

\subsection{Stylised facts of electricity prices}

The unique features of electricity cause its special price dynamics, in a literature also called the stylised facts (Girish et al., 2013). Note that some of these characteristics are observable not only for the spot prices, but also for their returns. The most visible is hourly, daily, weekly and annual seasonality caused by the climate changes and social factors that influence the consumption. Furthermore, these seasonal patterns depend on the climate location, i.e. in the countries closer to the equator the highest consumption is observed in the summer season due to airconditioning and in the countries with higher latitudes, the highest consumption occurs in winter months due to heating.

Extreme volatility which tends to form clusters is visible as well. It is caused by nonstorability of electricity, so in the unexpected change of consumption there are almost no reserves that can be used immediately. Electricity prices are also wellknown to exhibit the so-called spikes, which are rapid changes in price that can be both positive and negative with a slow reversion to the smaller/higher price. The reasons are the demand or supply shocks, transmission constraints, etc.

Apart from that, spot electricity prices can under certain circumstances attain even negative values and this phenomenon is not uncommon to observe in time series data, e.g. as depicted on Figure 1. The reason is that it is more profitable to sell electricity for a negative price (in fact paying the buyer for electricity) than to shut down the generators and then restart them again. Furthermore, as mentioned in the introductory part, electricity prices are assumed to be mean-reverting. Hence, they tend to move towards the long-term price level over some time that corresponds to the slowly changing marginal costs. 


\subsection{Electricity prices formation}

Formation of the spot (day-ahead) electricity prices is well-known and can serve as the starting point into the analysis of the formation of intraday prices of electricity. In general, from the economic theory the spot electricity price is established in the intersection of the demand and supply curves. The demand is highly inelastic at the given time and the supply curve (also called the merit order curve) represents the marginal costs of power generation using different power sources. Thus, electricity price represents the marginal cost of the last (with the largest marginal cost) power generating unit that wishes to participate in the electricity trading auction, so the spot electricity prices reflect several fundamental factors, which have an impact on the supply and demand for electricity. The most important factor is the weather conditions that influence the supply side via renewables and the consumption (demand) via air-conditioning, heating, etc. Another important demand-side factor is represented by the human-based business cycles such as peak-hours during the working day, weekends and holidays that cause the apparent seasonality. The supply-side factors consist of the finance-like variables such as the cost of building a power plant, its maintenance, operational costs and costs of the fuel used in non-renewable electricity plants, i.e. coal, natural gas, nuclear fuel prices, etc. Moreover, the spot electricity prices also reflect the impact of regulations such as Carbon Emission Allowance, which has an impact on the thermal power plant generation.

Prior to the analysis of the factors that influence the intraday electricity prices, we take a look at the intraday market mechanics in Czechia. In general, the intraday market organised by OTE complements the day-ahead market for trading throughout a day in the case of unpredicted events such as unexpected consumption, weather conditions, etc. The day-ahead market closes at $11 \mathrm{a} . \mathrm{m}$. on the day before any physical delivery takes place, and before 2:30 p.m. on the Day 1 the spot electricity prices are set for every hour of the Day 2 based on the expected demand (both domestic and exported/imported) for electricity and the forecasted load. In addition, the spot prices must take into account the cross-border flow between the countries, since the Czech, Slovak, Hungarian and Romanian day-ahead markets are interconnected.

The Czech intraday market opens daily at 3 p.m. and closes separately for each hourly period 60 minutes before the physical delivery. Since the intraday market participants have knowledge of the day-ahead price for the given hour, the intraday price for the exact hour should also reflect the improved forecast about the renewable production as a result of the updated weather forecast, updated knowledge of the demand, information about the power plants outages and updated electricity flow between countries as a result of the market coupling. Furthermore, it works the other way around; the intraday prices have impact on the day-ahead 
price on the next day. In a study on the differences between intraday and day-ahead electricity prices in the Danish power market, Karanfil et al. (2017) concluded that a difference between intraday and day-ahead prices is primary caused by the wind and conventional generation forecast errors and the intraday prices are negatively influenced by the forecast error of wind power generation. In addition, an unexpected increase in the combined heat and power generation leads to higher intraday prices and wider gap between intraday and day-ahead prices.

\section{Hurst Exponent and Time Series Predictability}

To conduct our research, we used a common measure of time series predictability the Hurst exponent, introduced by H. E. Hurst to study the long-term water levels in Aswan water reservoir (Hurst, 1951). It quantifies the long-term memory and relates to the autocorrelation of time series. Its estimation was originally based on the so-called Rescaled Range approach which assumes time series to be stationary, which might not be the case of most of the real financial time series. Thus, we used another well-known estimator of the Hurst exponent called the Detrended Fluctuation Analysis (described below), which is capable of handling both stationary and non-stationary (both in mean and variance) time series.

The Hurst exponent can take any value in the range from zero to one. Anti-persistent or mean-reverting time series have the Hurst exponent less than 0.5. The Hurst exponent for persistent time series is larger than 0.5 and the uncorrelated random time series have the Hurst exponent around 0.5.

This paper focuses on a common estimation algorithm which is called the Detrended Fluctuation Analysis (DFA) introduced by C. K. Peng (Peng et al., 1994) while studying the long-range correlations in DNA sequences. DFA is based on dividing the time series with $N$ observations into $k$ non-overlapping subsamples/windows with equal size $t$. Subsequently, within each subsample we find a local trend $y_{t}(k)$, which is a polynomial fit of some order. The linear fit is sometimes denoted as DFA(1), quadratic fit as DFA(2), etc. The integrated time series $y(k)$ is in turn detrended by subtracting the local trend in each subsample, and eventually we calculate the Fluctuation function for the specific time scale, defined as follows:

$$
F(t)=\sqrt{\frac{1}{N} \sum_{k=1}^{N}\left(y(k)-y_{t}(k)\right)^{2}}
$$

This calculation is repeated over all time scales to quantify the relationship between the scaling or fluctuation function $F(t)$ and the time-scale $t$ visible as the slope of the line between $\log F(t)$ and $\log (t)$, which corresponds to the scaling exponent $h$ as the estimate of the Hurst exponent if the time series is stationary. It is exponent because 
the relationship between $F(t)$ and the time scale $t$ ( $C$ is some constant) can be also written as:

$$
F(t)=C t^{h}
$$

The scaling exponent is larger than one for non-stationary time series and relates to the classical Hurst exponent simply as:

$$
H=h-1
$$

The Hurst exponent relates to the fractal or Hausdorff dimension for self-similar time series, since self-similar dynamic price processes are characterised by a reflection of the local structure into the global structure (Mandelbrot, 1985):

$$
D=2-H
$$

It quantifies the roughness or smoothness of time series, so it is a local (short-term memory) characteristic of time series as opposed to the Hurst exponent (Gneiting et al., 2004).

The application of the Hurst exponent can be extended to study the multifractal properties of time series using the Generalised Hurst exponent $H(q)$, where $q$ is the moment order of fluctuation function. Analogously, the so-called Multi-Fractal Detrended Fluctuation Analysis method (Kantelhardt, 2002) has been developed to estimate the values of the Hurst exponents for the given $q$. Then the scaling law takes the following form:

$$
F(q, t)=C t^{h(q)}
$$

For monofractal time series the fluctuation function is independent of $q$, and in the special case if $q=2$, the generalised Hurst exponent is equal to the classical Hurst exponent, which we deal with in this paper.

\section{Data and Methodology}

All calculations in this paper are based on the public data provided by the OTE, a.s. - the Czech electricity and gas market operator on its webpage (http://www.otecr.cz/statistika/) in the form of annual reports with comprehensive information about electricity trading. Nevertheless, for our purposes we used the average electricity prices on intraday trading with hourly frequency in EUR/MWh. To assure an adequate length of the dataset, our analysis was made on the data that spanned from 1.1.2017 to 31.12.2018 which totals in 17,520 observations.

In our research, we were interested in quantifying the Hurst exponent of the returns (increments) of actual prices after some variance-stabilising transformation as the majority of the related papers does due to the fact that intraday prices exhibit high volatility and spikes which impact not only the modelling (Uniejewski et al., 2017), 
but also the estimation of the Hurst exponent. However, calculation of the logreturns from electricity prices is not feasible when applied on negative or close to zero prices. There are some naïve methods of how to tackle these obstacles, for instance adding a constant to every observation, so it would be strictly positive (Sewalt et al., 2003) or dropping the negative observations. We tried, at first, to calculate the adjusted log-returns of the intraday electricity prices with naïve adjustment for the negative prices. Since the log-returns are quantifiable only on series with positive prices, we replaced 192 negatives out of 17,519 observations with zeros to get adjusted log-returns. Then we employed a different approach to calculate the returns using the area (inverse) hyperbolic sine transformation as suggested by Schneider (2012), because it shares some similarities with a logarithm and allows for zero and negative values:

$$
x=\sinh ^{-1}\left(\frac{p-\xi}{\lambda}\right)
$$

where $p$ is price, $\xi$ is an offset and $\lambda$ stands for scale. Due to simplicity, the offset is equal to zero and the scale is equal to one. The asymptotic log behaviour for $|p| \rightarrow$ $+\infty$ can be described as follows:

$$
x=\sinh ^{-1}(p)=\ln \left(p+\sqrt{p^{2}+1}\right) \approx \operatorname{sign}(p) \ln (2|p|)
$$

To calculate the Hurst exponent that varies with time, we decided to apply the socalled rolling or sliding window approach, which means that the calculation was made on a subsample with a size $N_{s}$ out of the total sample $N_{\max }$ called the sliding window. On each window, which was moved by defined step $\delta_{s}$, we calculated a static Hurst exponent to generate a sequence of Hurst exponents with length ranging from one when the subset coincides with the whole set to $N_{\max }-N_{s}+1$ if $\delta_{s}=1$. There is no strict consensus about how wide the rolling window should be. Nevertheless, we based our selection of the sliding window size on Carbone et al. (2004) who suggest that the minimum size of the subset should be $N_{\min } \approx 2,000$ 3,000 to achieve that the scaling law equation (2) holds.

Since we dealt with the hourly data, we experimentally chose the step to be 24 hours/observations (one day) and the sliding window to have four distinct lengths: 2,190 hours (three months), 4,380 hours (six months), 6,570 hours (nine months) and 8,760 hours (one year).

Consequently, the Hurst exponent sequences had a corresponding length, i.e. 638 days for three-month window, 547 days for six-month window, 456 days for ninemonth window and 365 days for a year-long window. 
Fig. 1: Intraday prices, logarithmic and inverse hyperbolic sine returns
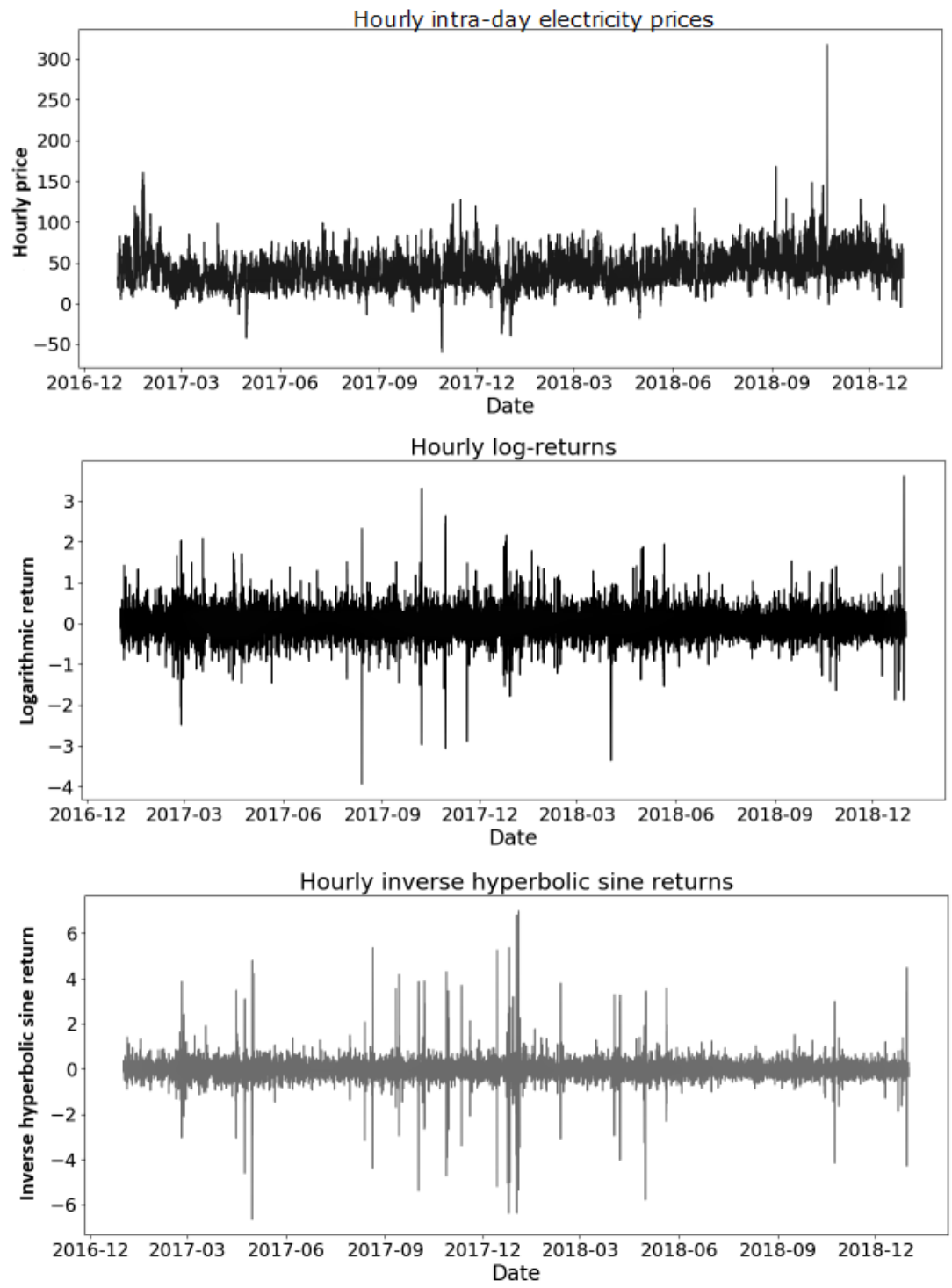

Source: Own computation, data from OTE webpage

\section{Results and Discussion}

We estimated the Hurst exponent by applying DFA method on the adjusted logreturns and inverse hyperbolic sine returns of hourly intraday prices of electricity using the statistical language R (RStudio ver. 1.1463, package nonlinearTseries ver. 
0.2.5). As the graphical results, we present plots of scaling function vs. scaling window size applied on a full sample to check for the potential crossovers, and the final plots of time evolution of the Hurst exponent estimated on the sliding windows of different sizes. Note that the DFA method does not take into account the window sizes below 10 due to its unreliability.

\subsection{Adjusted log-returns}

Applying the DFA method on the full sample of adjusted log-returns results in the Figure 2 on next page which clearly indicates at least two crossovers - change in the scaling exponents represented as the slope of the regression line. Therefore, we have three different Hurst exponents for three different time scales. However, since the fluctuation function estimations above the second crossover are evidently biased, we considered only time scales up to the second crossover. Thus, we calculated only two Hurst exponents.

Based on the graphical analysis and on the expert estimate, the position of the first crossover is located at approx. 24 hours, with the corresponding Hurst exponent being approx. 0.44, which indicates a weak mean-reverting process. The next crossover is less pronounced than the first one and located at the time scale of approx. 240 hours with the corresponding Hurst exponent being approx. 0.19, which indicates a stronger mean-reversion process than on the shorter time scales. This clearly shows that the log-returns of intraday electricity prices are not selfaffine since they behave differently on different time scales.

The interpretation follows from the mechanics of the intraday market described in Section 2.2. The intraday prices and their respective log-returns separated by one day are an outcome of the price formation process based on unexpected events, imperfectly forecasted power generation of renewables, load, and thereby consecutive (or the same) day-ahead prices. Since the day-ahead prices are also updated based on the previous intraday prices, for small time scales these updates are relatively recent. These unexpected effects project themselves in more randomlike behaviour of the intraday prices and returns, since the Hurst exponent close to 0.5 indicates the uncorrelated random process. On the higher time scales, the time-separation between the intraday prices is more than one day and thus their values are based on the aforementioned forecasted variables for different days. Consequently, the prices and their returns more likely tend to behave according to the stylised facts of the spot electricity prices, so they are mean-reverting. 


\section{Fig. 2: Estimation of two Hurst exponents on different time scales}

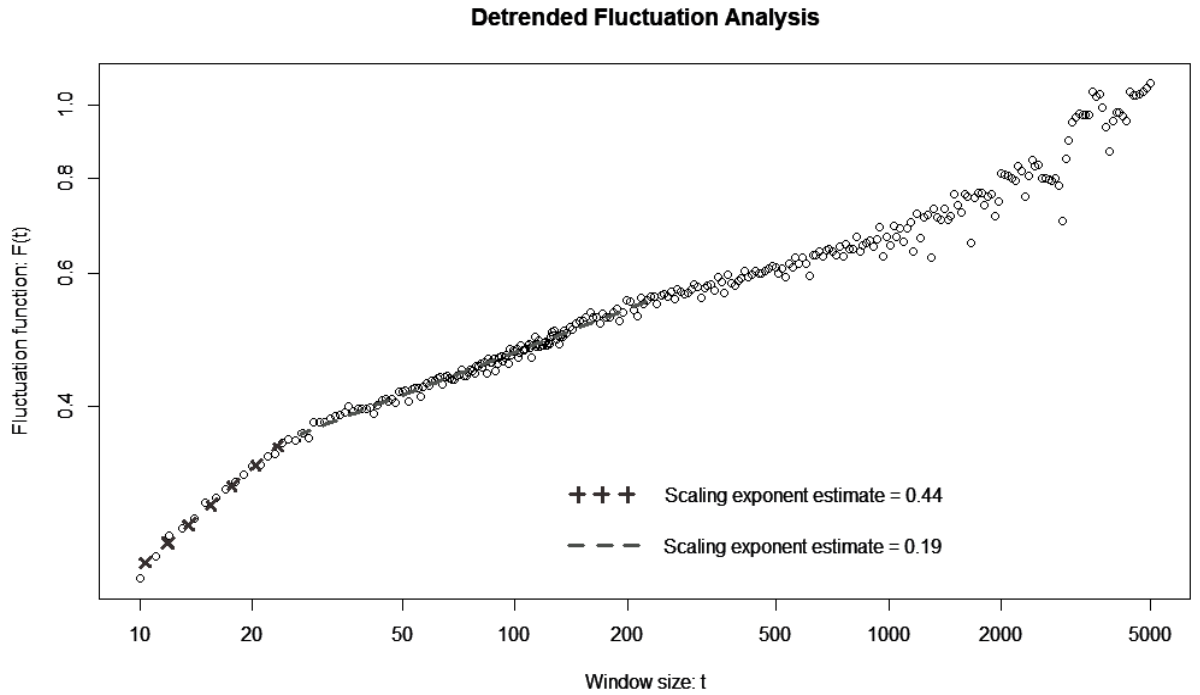

Source: Authorial computation

Due to the crossovers we estimated the time-varying Hurst exponents on two time scales - 10-24 hours and 25-240 hours, applying four different window sizes and a constant time step of 24 hours.

As depicted on Figures 3 and 4 on the next pages, in general, a wider sliding window smooths the sequence of Hurst exponents compared to the narrower one regardless of the time scale, and thereby it points out that the Hurst exponent stays quite stable when calculated on the year-long sample. On the other hand, in case of the shorter time scales, the sliding window with the width of 2,190 observations better captures dynamics of the estimated Hurst exponent with more details. Most of the time it indicated a weakly anti-persistent process, with a few exceptions when it revealed approximately random or even slightly persistent process for 53 out of 639 observations with $H_{\max }=0.56$ for the centre of the sliding window corresponding to the end of the year of 2017 and beginning of the 2018 .

On the longer time scales, the estimated Hurst exponent indicates a mean-reverting process for the whole time series and its dynamics is better visualised when using the narrower sliding window. Moreover, when using the year-long sample, the estimated Hurst exponent is roughly stable. The only sharp decline of the estimated Hurst exponent level is approximately for the last 50 values. 
European Financial and Accounting Journal, 2019, vol.14, no. 3, pp. 25-44.

Fig. 3: Hurst exponent - time scale of 10-24 hours
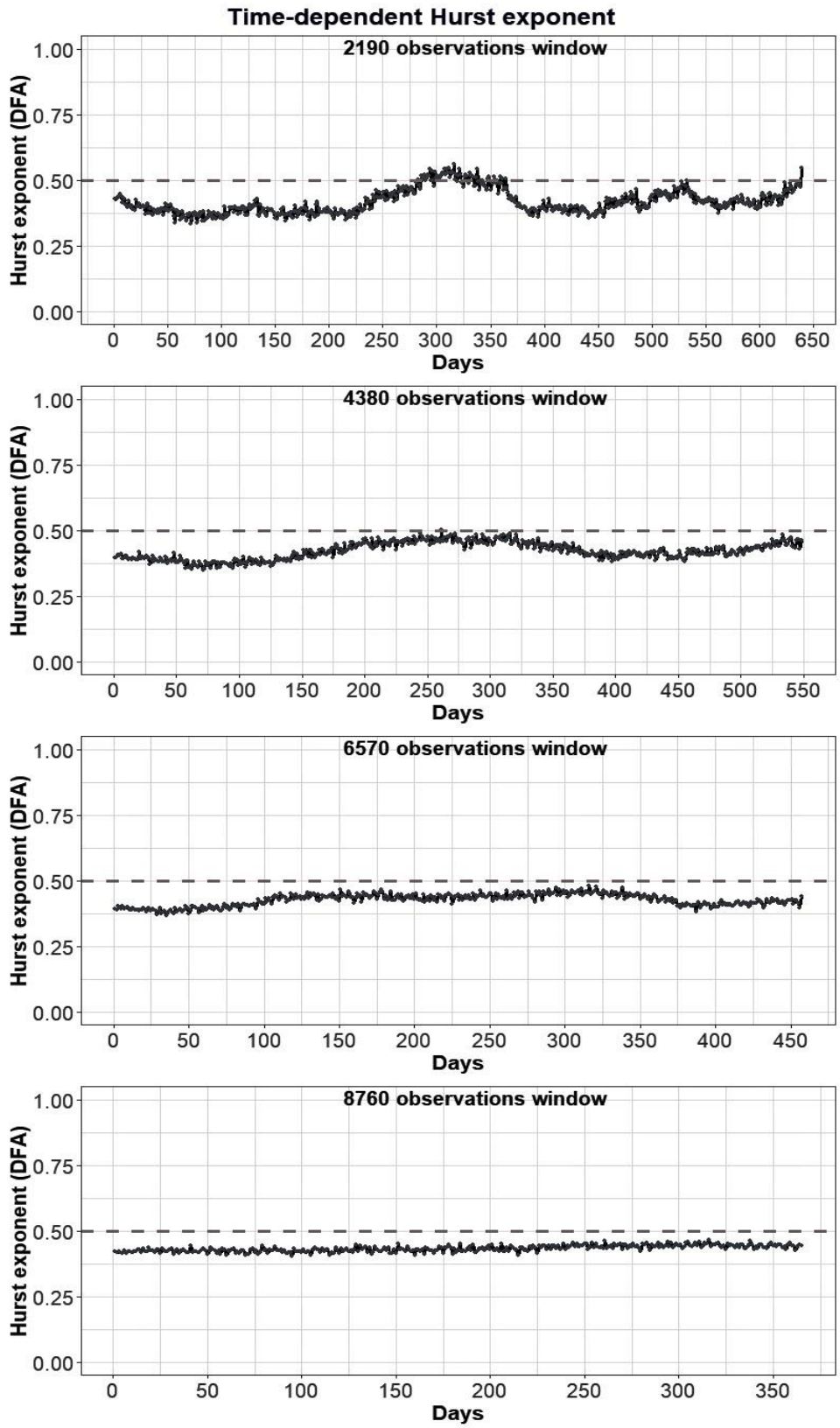

Source: Authorial computation 
Čurpek, J.: Time Evolution of Hurst Exponent: Czech Intraday Electricity Market Study

Fig. 4: Hurst exponent - time scale of 25-240 hours
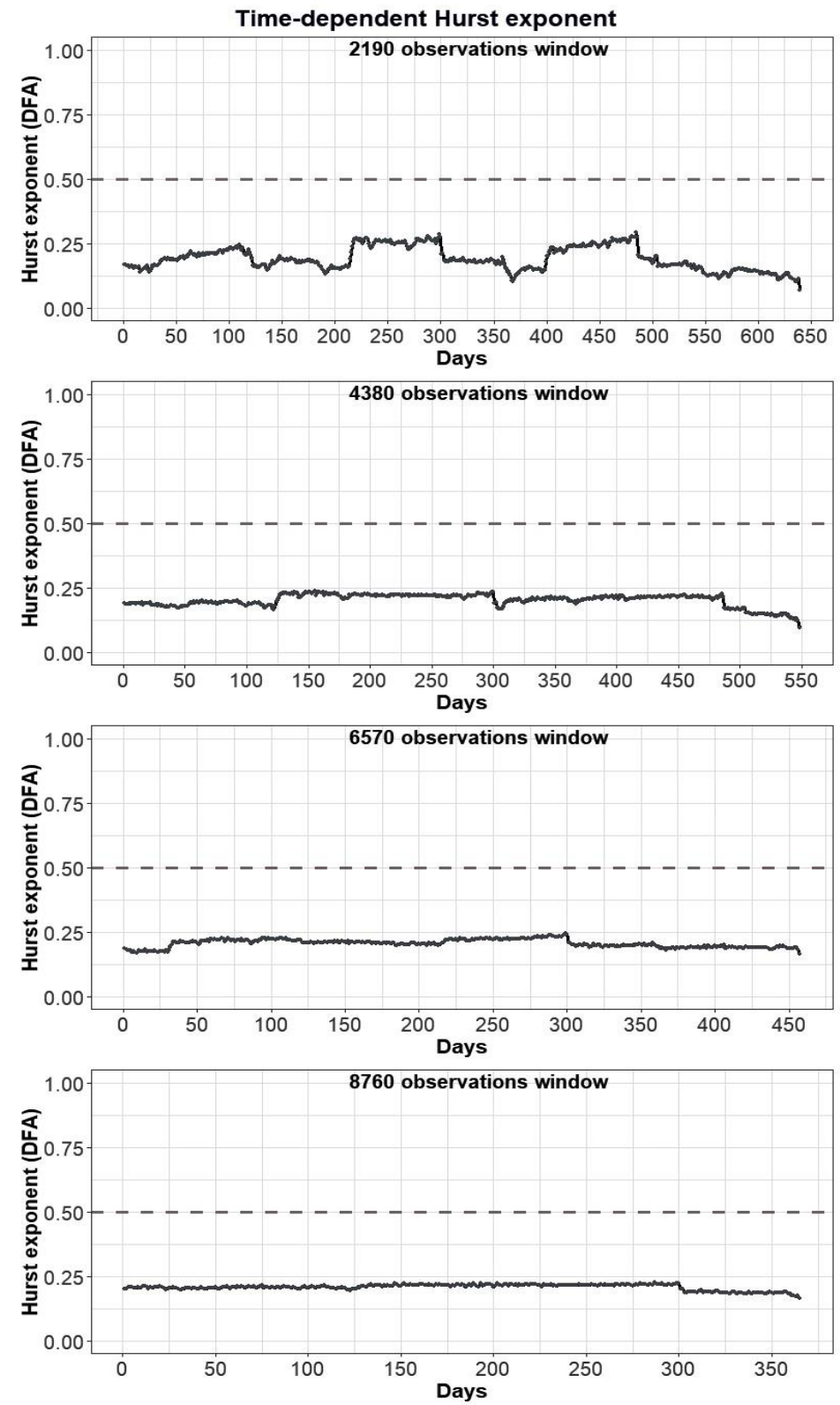

Source: Authorial computation 
Tab. 1: Average Hurst exponent

\begin{tabular}{ccccc}
\hline $\begin{array}{c}\text { Time scale/window } \\
\text { size }\end{array}$ & $\mathbf{2 , 1 9 0}$ & $\mathbf{4 , 3 8 0}$ & $\mathbf{6 , 5 7 0}$ & $\mathbf{8 , 7 6 0}$ \\
\hline $\mathbf{1 0 - 2 4} \mathbf{h}$ & 0.4186 & 0.4217 & 0.4260 & 0.4328 \\
$\mathbf{2 5 - 2 4 0 h}$ & 0.1913 & 0.2018 & 0.2059 & 0.2077 \\
\hline
\end{tabular}

Source: Authorial computation

\subsection{Inverse hyperbolic sine returns}

In case of inverse hyperbolic sine returns, the results are similar to those when using the adjusted log-returns. There are also two crossovers after applying the DFA method on the full-size sample of time series, as illustrated on Figure 5. Thus, we estimated two different Hurst exponents on two different time scales. The crossovers were again determined graphically and by expert estimate.

The first crossover again corresponds to approx. 24 hours with the estimated Hurst exponent being approx. 0.45, which indicates a weak mean-reversion. Next crossover is more noticeable than in the previous case (adjusted log-returns) and it is located at the time scale of approx. 240 hours. The respective estimated Hurst exponent at these time scales is approx. 0.19 which signifies a stronger meanreversion than on shorter time scales. Since estimated values of the fluctuation function are evidently biased on the higher time scales, we omitted the calculation of the Hurst exponent above the second crossover as in the case of log-returns. The interpretation of the different estimated Hurst exponents is the same as in the case of adjusted log-returns.

\section{Fig. 5: Estimation of two Hurst exponents on different time scales}

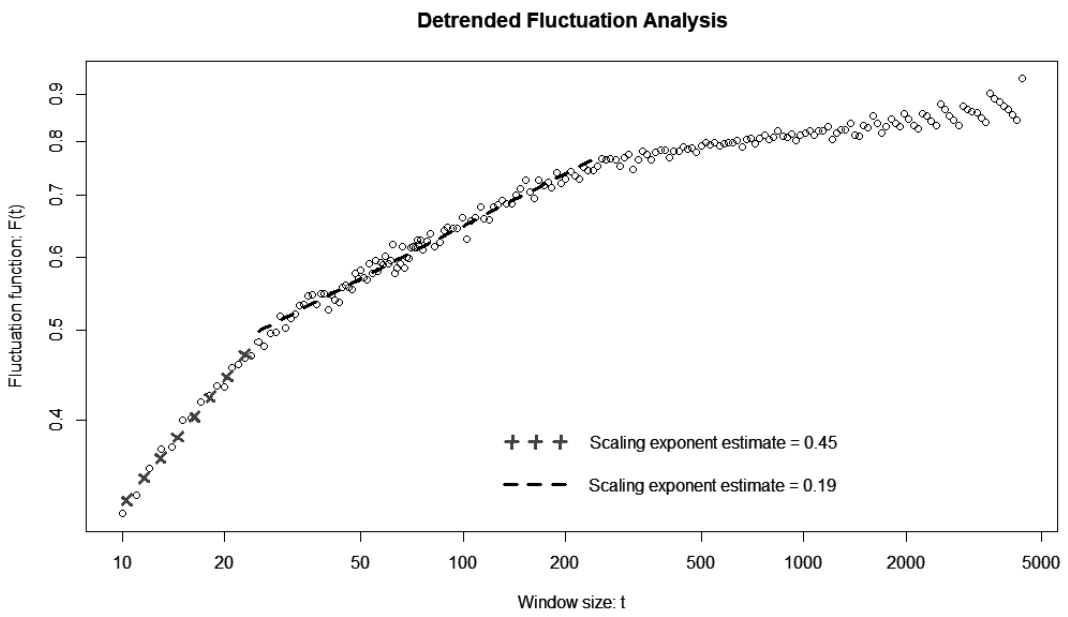

Source: Authorial computation 
The time-varying Hurst exponents were again computed using four different window sizes. The results are summarised in Table 2 and on Figures 6, 7.

In general, the results are analogous to the previous case when we worked with adjusted log-returns. Thus, the wider sliding window generates smoother sequence of estimated Hurst exponents compared to the narrower one, and the estimated Hurst exponent stays stable when calculated on the year-length sample for short time scales. For longer time scales, however, even using the sliding window of yearly length results in a sequence of estimated Hurst exponents that exhibits a level drop near the end, as revealed on Figure 7.

Similarly, in case of the short time scales, the sliding window with the width of 2,190 observations displays the estimated Hurst exponent attaining values associated with mean-reverting behaviour. However, in some cases the estimated Hurst exponent is even associated with almost random process or weakly persistent behaviour with $H_{\max }=0.55$. As illustrated on Figure 6, these events occur for sliding windows whose centre corresponds to the three different periods - approx. 06/2017, the period ranging from $12 / 2017$ to $01 / 2018$ and $06 / 2018$.

In addition, there is evident tendency of the estimated Hurst exponent rising almost to the level of 0.5 . The reason was explained in the previous section, i.e. the intraday electricity market should cover the unexpected and unforecasted changes in electricity demand and supply (generation) that was not reflected in the day-ahead prices. In case of short time scales, updates of these changes are relatively "fresh", since also the day-ahead prices and their returns are based on intraday prices, which reflect the changes of forecasted electricity variables. Thus, the imperfect forecast of the renewable generation load and sudden change of the expected demand (together with market participants' expectations) cause the intraday prices and their returns to behave in a more random-like way.

On the Figure 7 we noticed that on the longer time scales the estimated Hurst exponent indicates a mean-reverting process for the whole time series and its dynamics is better visualised when using the narrower sliding window.

\section{Tab. 2: Average estimated Hurst exponent}

\begin{tabular}{ccccc}
\hline Time scale/window & $\mathbf{2 , 1 9 0}$ & $\mathbf{4 , 3 8 0}$ & $\mathbf{6 , 5 7 0}$ & $\mathbf{8 , 7 6 0}$ \\
\hline $\mathbf{1 0 - 2 4}$ h & 0.4218 & 0.4284 & 0.4332 & 0.4411 \\
$\mathbf{2 5 - 2 4 0 h}$ & 0.1854 & 0.1995 & 0.2006 & 0.2001 \\
\hline
\end{tabular}

Source: Authorial computation 
European Financial and Accounting Journal, 2019, vol.14, no. 3, pp. 25-44.

Fig. 6: Hurst exponent - time scale of 10-24 hours
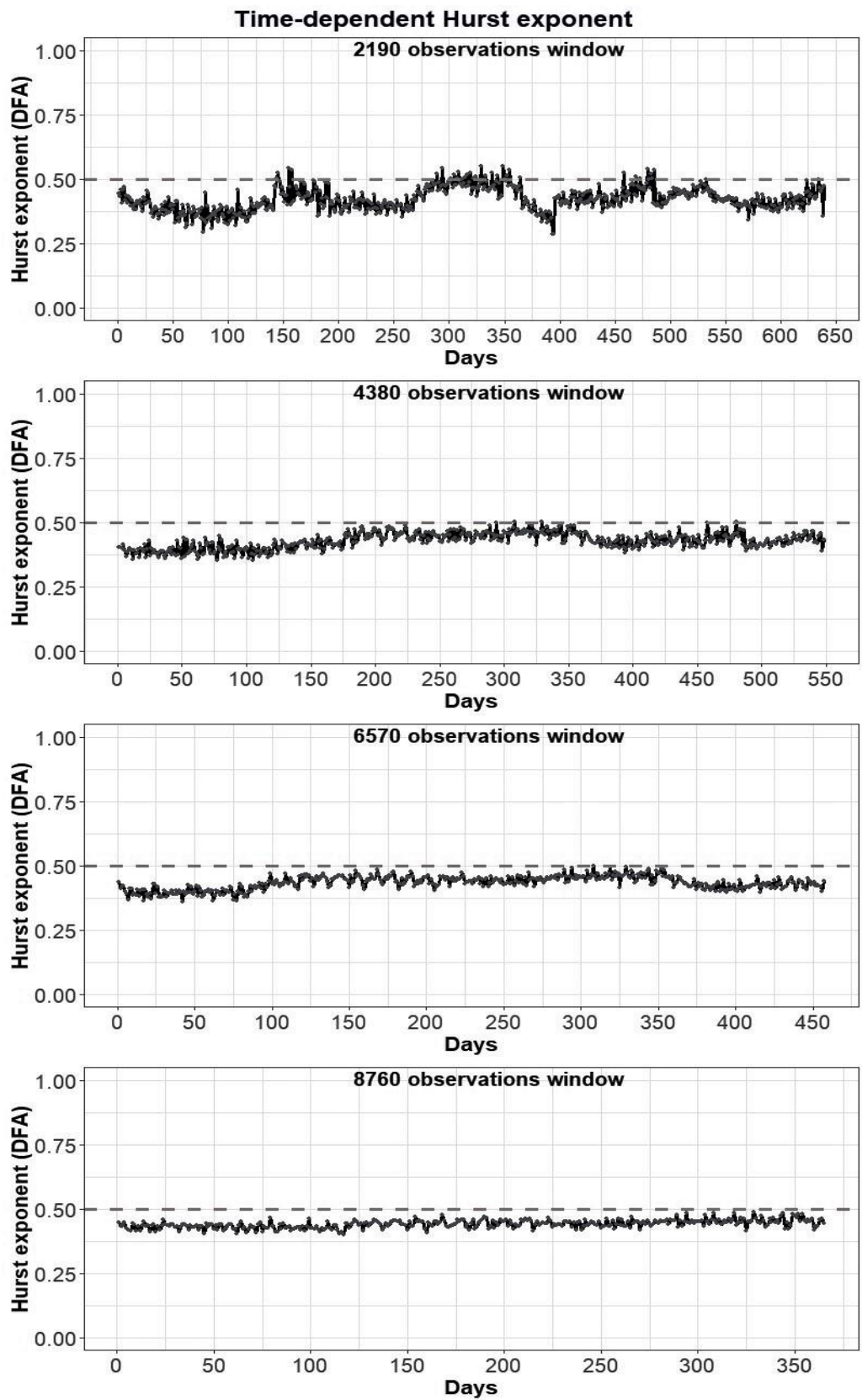

Source: Authorial computation 
Čurpek, J.: Time Evolution of Hurst Exponent: Czech Intraday Electricity Market Study

Fig. 7: Hurst exponent - time scale of 25-240 hours
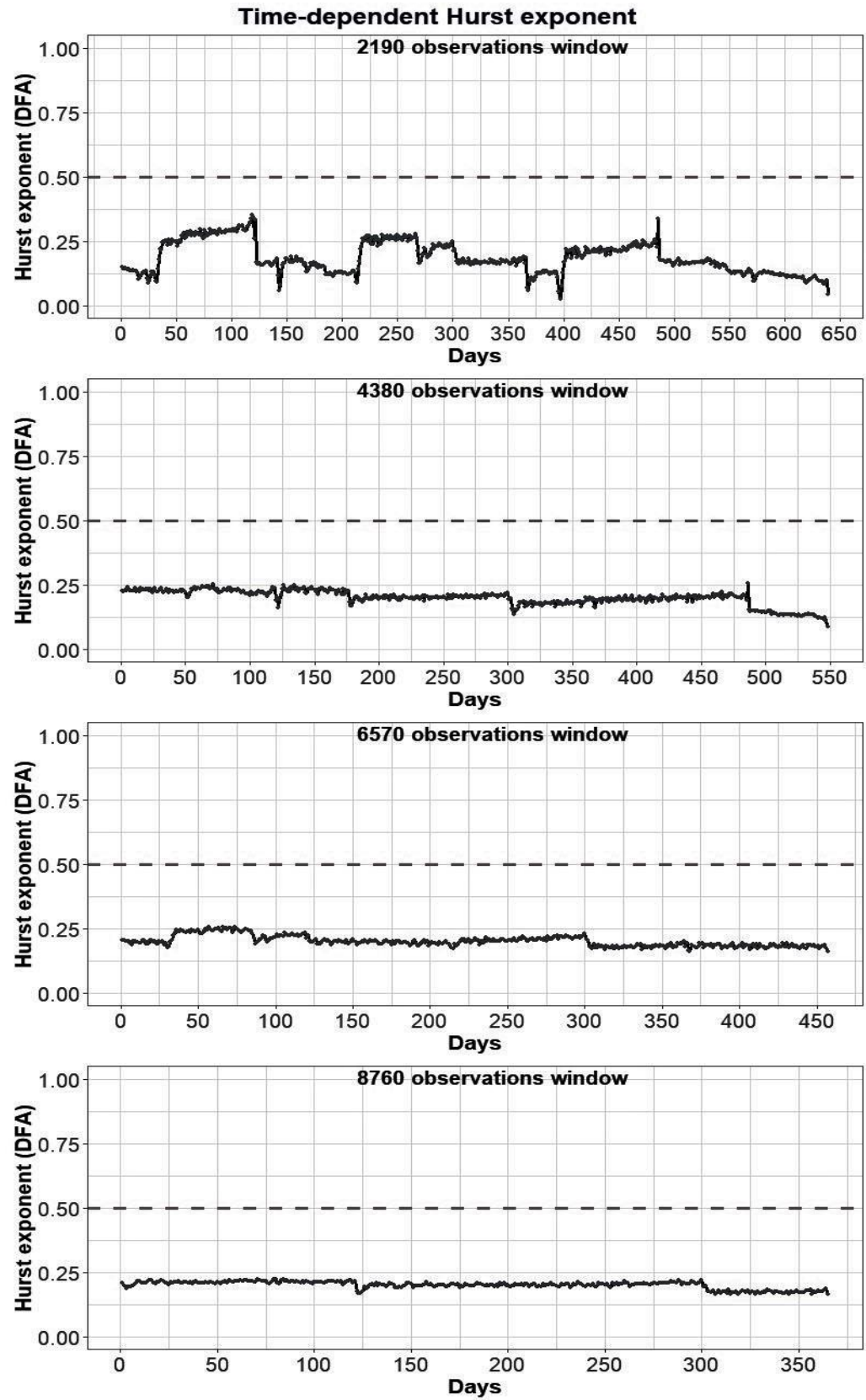

Source: Authorial computation 


\section{Conclusion}

In this paper we applied the Hurst exponent analysis on the Czech intraday electricity market, which still remains to be mostly unexplored. We employed the Detrended Fluctuation Analysis to estimate the time-varying Hurst exponent on hourly log-returns of the intraday electricity prices adjusted for the negative values, and on hourly area (inverse) hyperbolic sine returns. Due to presence of the crossovers in the scaling function, we concluded that the intraday hourly returns in our sample were not self-affine. Thus, on two different time scales divided by the crossover that corresponds to 24 hours, there are two different Hurst exponents. We assume that this is due to the intraday market price formation process, i.e. the intraday market complements the day-ahead market, which repetitively sets the prices (and load) on the daily basis for the whole next day. Thus, on the time scales of less than 24 hours, the intraday prices and returns are based on the unexpected supply and demand changes that were not forecasted by the dayahead market for the time interval between 12 and 36 hours in advance. Their time distance to their consecutive day-ahead forecast is the same for the time scale of 24 hours and less for smaller time scales. Moreover, the day-ahead prices and their returns are also based on the previous intraday prices, which are based on the updated forecast of the supply and demand changes. This mutual updating to the changes of fundamental factors that influence electricity prices causes the intraday returns on smaller time scales to be weakly mean-reverting or behave in a nearrandom way. However, the intraday prices and returns on the time scales above 24 hours are based on different, unexpected supply and demand changes from the dayahead market forecast, because they were made on more distant days. As a result, on the larger time scales the intraday returns behave more like the day-ahead returns, which are mean-reverting according to the stylised facts.

To get a better view of temporal evolution of the estimated Hurst exponent, we applied the sliding windows of different sizes on the both types of returns. They brought similar results as visualised by the plots, when the larger sliding windows caused larger smoothing of the estimated Hurst exponent sequence. Thus, to detect some dynamics in time we suggest not to use an excessively wide window, even though it might lead to more precise estimation of the Hurst exponent. In our research, the sliding window with a size of three months was satisfactory.

Moreover, we have come to the conclusion that the estimated Hurst exponent on both types of returns indicates that, in general, the intraday electricity returns are mean-reverting. For larger time scales above one day, the average value of the estimated Hurst exponent was approximately 0.2 regardless of the type of returns we used. This result is in agreement with the stylised facts of electricity prices (and also returns), now extended to intraday electricity returns. 
However, as the time scales get shorter than one day, the estimated Hurst exponent was associated with a weakly mean-reverting process, since the average value of the Hurst exponent was approximately 0.43 . Furthermore, in some short periods the estimated Hurst exponent was associated with a behaviour of the returns that is close to randomness or even weak persistence. In addition, using the inverse hyperbolic sine returns revealed that these nearly random or weakly persistent regimes occurred in the summer of 2017 and 2018 and in the winter between 2017 and 2018. Thus, these periods were separated by roughly 6 months. We believe these were caused by the imperfect forecast of mainly renewable generation load (dependent on the weather) and a sudden change of the expected demand together with the expectations of the market participants, which substantially deviated from the real (generated) supply and demand. Thus, a more precise forecast of the power production, especially of the renewable sources, which are based on the weather forecast, together with the expected demand would diminish the gap between the intraday and day-ahead prices and their returns.

Further research into the behaviour of the Hurst exponent on the intraday electricity market with data comprising several years may show if these periods of non-meanreverting or almost random behaviour can be detected on different markets and for different periods.

\section{References}

Bergh, Van den, K., Boury, J., Delarue, E., 2016. The Flow-Based Market Coupling in Central Western Europe: Concepts and definitions. The Electricity Journal, 29(1), 24-29, 2016. DOI: 10.1016/j.tej.2015.12.004.

Béreš, S., 2017. Impact of Czech intraday market on the electricity prices. Bachelor thesis. Charles University. Faculty of Social Sciences Institute of Economic Studies. Carbone, A.; Castelli, G.; Stanley, H.E., 2004. Time-dependent Hurst exponent in financial time series. Physica A: Statistical Mechanics and its Application, 344:267-271. DOI: 10.1016/j.physa.2004.06.130.

Girish, G. P., Vijayalakshmi S., 2013. Determinants of Electricity Price in Competitive Power Market. International Journal of Business and Management; Vol. 8, No. 21. Canadian Center of Science. DOI: 10.5539/ijbm.v8n21p70.

Gneiting, T., Schlather, M., 2004. Stochastic models that separate fractal dimension and the hurst effect. SIAM Review. 2004; 46:269. DOI: 10.1137/S0036144501394387.

Hong, T., Fan, S., Pinson, P., Zareipour, H, Troccoli, A., Hyndman, R. J., 2016. Probabilistic energy forecasting: Global Energy Forecasting Competition 2014 and beyond. International Journal of Forecasting 32, 914-938. DOI: 10.1016/j.ijforecast.2016.02.001. 
Hurst, H. E., 1951. Long-Term Storage of Reservoirs: An Experimental Study. Transactions of the American Society of Civil Engineers, 116, 770-799.

Kantelhardt, J., Zschiegner, S., Koscielny-Bunde, E., Bunde, A., Havlin, S., Stanley E., 2002. Multifractal Detrended Fluctuation Analysis of Nonstationary Time Series. Physica A: Statistical Mechanics and its Applications, 316(1-4):87-114.

Karanfil, F., Li, Y., 2017. The Role of Continuous Intraday Electricity Markets: The Integration of Large-Share Wind Power Generation in Denmark. Energy Journal, $38(2)$.

Kristoufek, L., Lunackova, P., 2013. Long-term Memory in Electricity Prices: Czech Market Evidence. Faculty of Social Sciences, Charles University in Prague, Available from: <journal.fsv.cuni.cz/storage/1282_407-24---kristoufek.pdf>. [22 June 2018].

Mandelbrot, B., 1985. Self-affine fractals and fractal dimension. Physica Scripta. 1985;32:257-260. DOI: 10.1088/0031-8949/32/4/001.

Meyer-Brandis, T., Tankov P., 2008. Multifactor jump-diffusion models of electricity prices. International Journal of Theoretical and Applied Finance, Vol. 11 (5) (2008), pp. 503-528. DOI: 10.1142/S0219024908004907.

Monteiro, C., Ramirez-Rosado, I., Fernandez-Jimenez, L., Conde, P., 2016. Shortterm price forecasting models based on artificial neural networks for intraday sessions in the Iberian electricity market. Energies 9 (9).

Peng, C.-K., Buldyrev, S.V., Havlin, S., Simons, M., Stanley, H.E., Goldberger, A.L., 1994. Mosaic Organization of DNA nucleotides. Physical Review E. 1994;49(2):1685. DOI: 10.1103/PhysRevE.49.1685.

Sewalt, M., De Jong, C., 2003. Negative Prices in Electricity Markets. Commodities Now (June 2003), pp. 74-77. Available from: <kyos.com/wpcontent/uploads/2016/10/Commodities-Now-Negative-prices-in-ElectricityMarkets.pdf>. [15 June 2019].

Schneider, S., 2011. Power spot price models with negative prices. The Journal of Energy Markets 4(4):77-102, December 2011, pp. 77-102.

Simonsen, I., 2003. Measuring anti-correlations in the Nordic electricity spot market by wavelets. Physica A: Statistical Mechanics and its Application, 322:597-606. DOI: 10.1016/S0378-4371(02)01938-6.

Uniejeweski, B., Marcjasz, G., Weron, R., 2019. Understanding intraday electricity markets: Variable selection and very short-term price forecasting using LASSO. International Journal of Forecasting. DOI: 10.1016/j.ijforecast.2019.02.001.

Uniejewski, B., Weron, R., Ziel, F., 2017. Variance stabilizing transformations for electricity spot price forecasting. IEEE Transactions on Power Systems, pp. (99):11. DOI: 10.1109/TPWRS.2017.2734563. 
Uritskaya, O. Y., Uritsky, V. M., 2015. Predictability of price movements in deregulated electricity markets. Available from: <arxiv.org/abs/1505.08117>. [20 June 2019].

Weron, R., Przybyłowicz, B., 2000. Hurst analysis of electricity price dynamics. Physica A: Statistical Mechanics and its Applications. 283. 462-468. DOI: 10.1016/S0378-4371(00)00231-4 